\title{
A Correlation Study of Clinical Outcomes by Quantification of Fatty Degeneration of the Subscapularis: Partial vs. Whole Cross-section
}

\author{
Joo Hyun Park, Kwang Yeol Lee, Sung Min Rhee, Joo Han $\mathrm{Oh}^{\circledR}$ \\ Department of Orthopaedic Surgery, Seoul National University Bundang Hospital, Seoul National University College of Medicine, Seongnam, ${ }^{1}$ Department of \\ Orthopaedic Surgery, Mega Hospital, Gimhae, Korea
}

Background: Fatty degeneration of rotator cuff is a well-known predictor of postoperative outcome. The purpose of this study was to evaluate the clinical features of rotator cuff tears involving subscapularis, and investigate whether fatty degeneration quantified from only the upper subscapularis correlates better with clinical outcomes than quantified from the whole subscapularis.

Methods: We retrospectively analyzed 315 consecutive patients who underwent arthroscopic repair for rotator cuff tears involving subscapularis with a minimum follow-up of 1 year. Preoperative and postoperative visual analogue score for pain, range of motion and functional scores were assessed. Integrity of the repaired tendon was assessed at the 1-year follow-up with either magnetic resonance imaging or ultrasonography.

Results: The mean Goutallier grade of whole cross-section was significantly lower than that of upper cross-section (1.59 vs. 1.71, $\mathrm{p}<0.05)$, but significantly higher than that of lower cross-section $(1.59$ vs. $1.01, p<0.05)$. In analysis of 37 re-tears, the occupancy of severe fatty degeneration in upper cross-section was $86.5 \%$, which was significantly higher than that seen in whole cross-section $(56.8 \%$, $\mathrm{p}$ <0.05). We calculated the cut-off tear size for prediction of re-tears as $19.0 \mathrm{~mm}$ for retraction and $11.0 \mathrm{~mm}$ for superior-inferior. The cut-off Goutallier grade was 2.5 for both whole and upper cross-sections, but area under the curve was greater in the upper cross-section than the whole $(0.911$ vs. 0.807$)$.

Conclusions: As fatty degeneration of upper subscapularis demonstrated a more distinct spectrum than whole subscapularis, we suggest that measuring fatty degeneration of upper subscapularis can be a more useful method to predict clinical prognosis.

(Clin Shoulder Elbow 2018;21(2):67-74)

Key Words: Rotator cuff tear; Subscapularis; Fatty; Degeneration

\section{Introduction}

The subscapularis muscle comprises of four to six collagen bundles. The superior bundle attaches to the medial wall of the biceps long head tendon, and the inferior bundle, which is shorter than the superior bundle, has been shown to be closer to the muscle layer than the superior bundle. ${ }^{1,2)}$ The upper and lower sides of the subscapularis have been shown to be controlled by different nerves. ${ }^{3)}$ Researchers have shown that lesions of the subscapularis generally come in one of the following three forms: 1) isolated tears; 2) combined tears of the coracohumeral ligament, the superior glenohumeral ligament, and the supraspinatus that are confined to the anterosuperior region; or 3) massive tears encompassing the infraspinatus muscle. ${ }^{4-6)}$ Recent studies have shown that subscapularis tears that invade more than half of the subscapularis are associated with a significantly higher rate of re-tear than those that invade less than half, suggesting the importance of subscapularis tears. ${ }^{7)}$

Fatty degeneration of the rotator cuff is a well-known predictor of clinical outcome. ${ }^{8)}$ Studies on the quantification of fatty

Received January 22, 2018. Revised March 19, 2018. Accepted March 26, 2018.

Correspondence to: Joo Han Oh

Department of Orthopaedic Surgery, Seoul National University Bundang Hospital, Seoul National University College of Medicine, 82 Gumi-ro 173beon-gil, Bundang-gu, Seongnam 13620, Korea

Tel: +82-31-787-7197, Fax: +82-31-787-4056, E-mail: ohjh1@snu.ac.kr, ORCID: https://orcid.org/0000-0002-4962-3175

IRB approval: Seoul National University Bundang Hospital (B-1801/445-107).

Financial support: None. Conflict of interests: None. 
degeneration as a prognostic marker are limited. Previous studies have analyzed the degree of fatty degeneration, for instance, by the type of supraspinatus tear, ${ }^{9}$ but none have investigated the effect of the area of muscle used to quantify the degree of fatty degeneration on the predictive value of the variable. Therefore, we investigated whether quantification of the degree of fatty degeneration with the upper cross-section of the subscapularis is a more accurate predictor of clinical outcome for subscapularis tears than that with the whole cross-section.

\section{Methods}

\section{Subjects of Study}

Between February 2010 and May 2015, we recruited patients in accordance to the following selection criteria: patients with a preoperative magnetic resonance imaging (MRI) diagnosis of a high-grade partial cuff tear or a full-thickness cuff tear involving the subscapularis; patients who underwent an arthroscopic subscapularis repair; patients who were followed-up for at least 1 year; and those who had an ultrasound or MRI examination at the 1-year follow-up to determine a successful repair. Patients who were lost to follow-up (83 patients); had a history of cuff repair or fractures (15 patients), which was assessed by using patient medical records or patient anamnesis; or had systemic inflammatory diseases, such as a rheumatoid arthritis (10 patients) were excluded. These inclusion and exclusion criteria led to a final sample of 315 patients in our retrospective study. The study population consisted of 151 men and 164 women, and the average age of the patients at the time of operation was 63.1 years (range, 49-81 years).

\section{Study Design}

Using sagittal MRI imaging, we measured the degree of fatty degeneration of the subscapularis muscle on the 'scapular $Y$ view', on which the intersection between the scapular spine and the scapular body could be seen. We quantified the degree of fatty degeneration on three cross-sections of the subscapularisupper, lower, and whole cross-sections. To divide the subscapularis tendon into its upper and lower halves, we first identified the upper and lower slopes extending from the most prominent anterior protrusion of the lesser tuberosity of the humerus on the MRI plane that shows the biceps long head tendon. Following the length of the fiber-bundle slopes leads to the scapular $\mathrm{Y}$ view, and the division between the two halves of the subscapularis
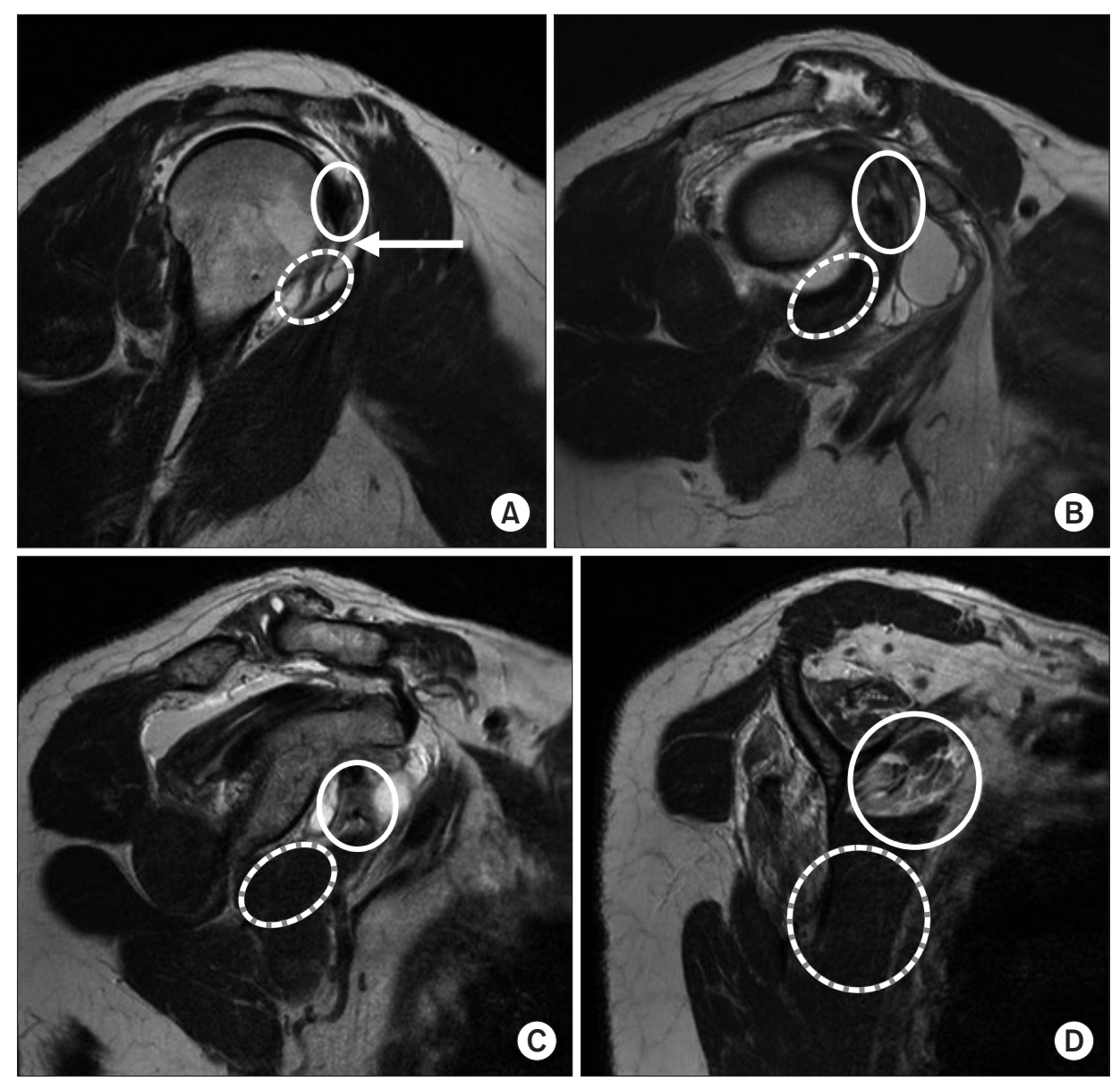

Fig. 1. (A-D) Images from the humerus towards the glenoid. The upper (circles), lower (dotted circles), and whole (arrow) crosssections of the subscapularis are shown. 
was made approximately $1-2 \mathrm{~cm}$ inferior to the center of this ' $\mathrm{Y}$ ' branch (Fig. 1).

To determine the postoperative outcome, we compared the preoperative values of the visual analogue score for pain (pVAS), the active range of motion (ROM), and functional parameters, the American Shoulder and Elbow Surgeons (ASES) score and the simple shoulder test (SST), to their respective postoperative values at the 1-year follow-up. We evaluated the re-tears with ultrasonography or MRI. To assess internal rotator strength, we used the isokinetic muscle performance test and the shoulder strength index (SSI; which measures muscle strength in relation to the unaffected contralateral side). We calculated the degree of fatty degeneration of the subscapularis in three cross-sections of the subscapularis - upper, lower, and whole. We investigated the relationship between tear size and the degree of fatty degeneration by the subscapularis cross-section quantified and whether these factors each affects the rate of postoperative retears.

\section{Statistical Analysis}

We performed all statistical analysis with Stata/SE 14 (StataCorp LP, College Station, TX, USA) and the open-source statistical software $\mathrm{R}$ version 3.3.2 (http://www.R-project.org). To compare the pre- and postoperative ASES score, SST, pVAS and ROM, we used the paired t-test and the Wilcoxon signed rank test. Statistical significance was measured in terms of the 95\% confidence level. We used the linear mixed model to assess the difference in the average level of fatty degeneration by subscapularis cross-section and one-way ANOVA to analyze the association between internal rotator strength, subscapularis tear size, and fatty degeneration by subscapularis cross-section. We used the chi-squared test to analyze the association between the type of subscapularis tear, the presence of trauma, the re-tear rate, and the level of fatty degeneration by subscapularis crosssection. To analyze our predictors of re-tear, the extent of retraction of subscapularis tears and cut-off values for fatty degeneration, we used the receiver operating characteristic curve.

\section{Results}

The average postoperative follow-up period was 32.7 months (range, 12-70 months). We found that the ROM at 1-year follow-up was significantly improved than the preoperative period. The forward flexion significantly improved from $143.17^{\circ} \pm$ $36.82^{\circ}$ preoperatively to $163.44^{\circ} \pm 26.83^{\circ}$ at the 1 -year followup; the external rotation significantly improved from $56.22^{\circ} \pm$ $18.30^{\circ}$ to $75.0^{\circ} \pm 16.47^{\circ}$, respectively; and the internal rotation significantly improved from thoracic vertebral level $10.49 \pm 3.01$ to $8.60 \pm 1.77$, respectively (all $p<0.05$ ). The average pVAS improved from $6.91 \pm 2.29$ points preoperatively to $0.82 \pm 1.67$ points at the 1-year follow-up. The functional scores, ASES and SST, also showed a significant improvement after surgery (47.01 \pm 17.71 to $91.19 \pm 13.73$ and $6.37 \pm 1.80$ to $10.07 \pm 3.19$, respectively; all $p<0.05$ ).

We classified the patients into three groups in terms of the type of tear: $6.7 \%$ of patients had isolated subscapularis tears (21 patients); $50.8 \%$ of patients had combined subscapularis and supraspinatus tears (160 patients); and $42.5 \%$ of patients had combined subscapularis, supraspinatus, and infraspinatus tears (134 patients). We found the presence of trauma history in $26.7 \%$ of patients (84 patients), and this proportion was particularly high in patients with isolated subscapularis tears, increasing to $61.9 \%$ in these subset of patients (13 of 21 patients). However, the type of tear and presence of trauma history each did not correlate with the degree of fatty degeneration of the subscapularis in any of the subscapularis cross-sections (all $p>0.05$ ).

We found that our indicator of internal rotator strength, SSI, decreased as the degree of fatty degeneration of the subscapularis became more severe. However, the correlation was not statistically significant for any of the cross-sections measured (whole cross-section $[p=0.378]$ and upper cross-section $[p=0.520]$ ).

In our sample of patients, we found that most lesions of the subscapularis were confined to the upper subscapularis $(72.9 \%)$. We also found that the size of subscapularis tear significantly correlated with the degree of fatty degeneration irrespective of the area of subscapularis measured (upper, lower, and whole crosssections) (all $p<0.05$ ) (Table 1 ). The degree of degeneration according to the Goutallier grading system was on average $1.59 \pm$ 0.06 for whole cross-sections; interestingly, this was significantly lower than that determined for upper cross-sections (1.71 \pm $0.06)(p<0.05)$ and significantly higher than that determined for lower cross-sections $(1.01 \pm 0.06)(p<0.05)$.

At the 1-year follow-up, the integrity of the repaired tendon was assessed through either MRI (142 patients) or ultrasonography (173 patients). We found that $11.7 \%$ of patients had subscapularis re-tears (37 patients). Unsurprisingly, we found that a patient's likelihood to have a re-tear was significantly associated with the degree of fatty degeneration the patient had; this pattern was observed irrespectively of the area of subscapularis quantified for fatty degeneration $(p<0.05)$ (Table 2). We found that the re-tear rate was markedly different between those with severe fatty degeneration (Goutallier grade 3-4) and those with mild fatty degeneration (Goutallier grades 0-2). Again, the difference in re-tear rate by the severity of fatty degeneration was observed irrespectively of the area of subscapularis measured. When whole cross-sections of the subscapularis were used to quantify the extent of fatty degeneration, we found that those classified as having severe fatty degeneration had a re-tear rate of $44.7 \%$ whilst those classified as having mild degeneration, $6.0 \%$. The respective values when upper cross-sections of the 
Table 1. Correlation between Fatty Degeneration and Tear Size of Subscapularis according to the Measurement Methods*

\begin{tabular}{lcc}
\hline \multicolumn{1}{c}{ Variable } & Retraction size $(\mathrm{mm})$ & Superior-inferior size $(\mathrm{mm})$ \\
\hline Fatty degeneration of SSC-W (Goutallier grade) & \\
\hline 0 & $10.86 \pm 3.21$ & $9.55 \pm 3.46$ \\
1 & $12.01 \pm 6.40$ & $10.67 \pm 5.01$ \\
\hline 2 & $16.18 \pm 7.97$ & $14.26 \pm 6.52$ \\
\hline 3 & $19.95 \pm 8.71$ & $18.90 \pm 8.10$ \\
4 & $22.35 \pm 11.28$ & $22.61 \pm 10.56$
\end{tabular}

Fatty degeneration of SSC-U (Goutallier grade)

$\begin{array}{llr}0 & 11.04 \pm 3.25 & 9.65 \pm 3.42 \\ 1 & 11.83 \pm 6.42 & 10.57 \pm 5.05 \\ 2 & 14.51 \pm 6.13 & 13.44 \pm 5.74 \\ 3 & 21.79 \pm 10.48 & 16.26 \pm 7.54 \\ 4 & 22.14 \pm 10.25 & 22.14 \pm 9.70\end{array}$

Fatty degeneration of SSC-L (Goutallier grade)

\begin{tabular}{lll}
0 & $11.82 \pm 5.09$ & $10.55 \pm 4.37$ \\
1 & $13.83 \pm 7.57$ & $12.31 \pm 6.11$ \\
2 & $18.33 \pm 6.83$ & $18.83 \pm 8.50$ \\
3 & $19.20 \pm 8.58$ & $19.20 \pm 8.55$ \\
4 & $32.83 \pm 13.42$ & $27.67 \pm 15.77$ \\
\hline
\end{tabular}

Values are presented as mean \pm standard deviation.

SSC-W: subscapularis on whole cross-section, SSC-U: subscapularis on upper cross-section, SSC-L: subscapularis on lower cross-section. ${ }^{*} p<0.05$.

subscapularis were used were $51.6 \%$ and $2.0 \%$ for severe and mild fatty degeneration, respectively. However, when the lower cross-sections were used, the re-tear rates were $57.1 \%$ and $9.6 \%$ for severe and mild fatty degeneration, respectively, suggesting that compared to the former two quantification methods, the latter method to quantify the degree of fatty degeneration leads to a relatively high rate of re-tears (40.6\%) even among patients with fatty degeneration of Goutallier grade 2 .

For patients who showed re-tears (37 patients), we classified them into different groups in terms of their degree of fatty degeneration. This grouping was performed for all three sets of fatty degeneration quantifications (from the upper, lower, and whole cross-sections). Fatty degeneration was classified as 'severe' for Goutallier grades 3-4 or 'mild' for Goutallier grades $0-2$. Our findings show that $86.5 \%$ of patients (32 patients) had severe fatty degeneration when the upper cross-section of the subscapularis was measured for fatty degeneration; this proportion was significantly higher than that determined when the whole cross-section of the subscapularis was taken (56.8\% [21 patients], $p<0.05)$. In contrast, $78.4 \%$ from the sample patient population (29 patients) were found to have mild fatty degen-
Table 2. Correlation between Fatty Degeneration and Retear of Subscapularis according to the Measurement Methods*

\begin{tabular}{|c|c|c|c|}
\hline & Healed case $(n)$ & Retear case (n) & Retear rate $(\%)$ \\
\hline \multicolumn{4}{|c|}{ Fatty degeneration of SSC-W (Goutallier grade) } \\
\hline 0 & 24 & 0 & 0 \\
\hline 1 & 146 & 6 & 3.9 \\
\hline 2 & 82 & 10 & 10.9 \\
\hline 3 & 13 & 7 & 35.0 \\
\hline 4 & 13 & 14 & 51.9 \\
\hline Total (n) & 278 & 37 & \\
\hline
\end{tabular}

Fatty degeneration of SSC-U (Goutallier grade)

\begin{tabular}{|cccc|}
\hline 0 & 25 & 0 & 0 \\
\hline 1 & 143 & 1 & 0.7 \\
\hline 2 & 80 & 4 & 4.8 \\
\hline 3 & 11 & 10 & 47.6 \\
\hline 4 & 19 & 22 & 53.7 \\
\hline Total $(\mathrm{n})$ & 278 & 37 & \\
\hline
\end{tabular}

Fatty degeneration of SSC-L (Goutallier grade)

\begin{tabular}{lrrr|}
0 & 62 & 3 & 4.6 \\
1 & 191 & 13 & 6.4 \\
\hline 2 & 19 & 13 & 40.6 \\
\hline 3 & 3 & 3 & 50.0 \\
4 & 3 & 5 & 62.5 \\
\hline Total (n) & 278 & 37 & \\
\hline
\end{tabular}

SSC-W: subscapularis on whole cross-section, SSC-U: subscapularis on upper cross-section, SSC-L: subscapularis on lower cross-section. ${ }^{\star} p<0.05$.

eration when the lower cross-section of the subscapularis was taken to quantify the degree of fatty degeneration. Given that there were no patients with tears or fatty degeneration only in the lower subscapularis, our findings suggest that quantification of fatty degeneration from only the lower cross-sections of the subscapularis cannot be a meaningul predictor for clinical outcome or prognosis.

We found that the cut-off values for our predictors of subscapularis re-tears, the size of retraction and the superior-inferior length was $19.0 \mathrm{~mm}$ (area under the curve $[\mathrm{AUC}]=0.784$ ) and $11.0 \mathrm{~mm}(\mathrm{~A} \cup \mathrm{C}=0.803$ ), respectively (Fig. 2). We also found that the cut-off value for the degree of fatty degeneration with predictive power for subscapularis re-tears was a Goutallier grade 2.5. The cut-off value was the same whether the degree of fatty degeneration was measured from whole cross-sections or from upper cross-sections of the subscapularis, but AUC was significantly higher for the latter than the former $(A \cup C=0.911$ vs. 0.807 , respectively). This suggests that fatty degeneration 

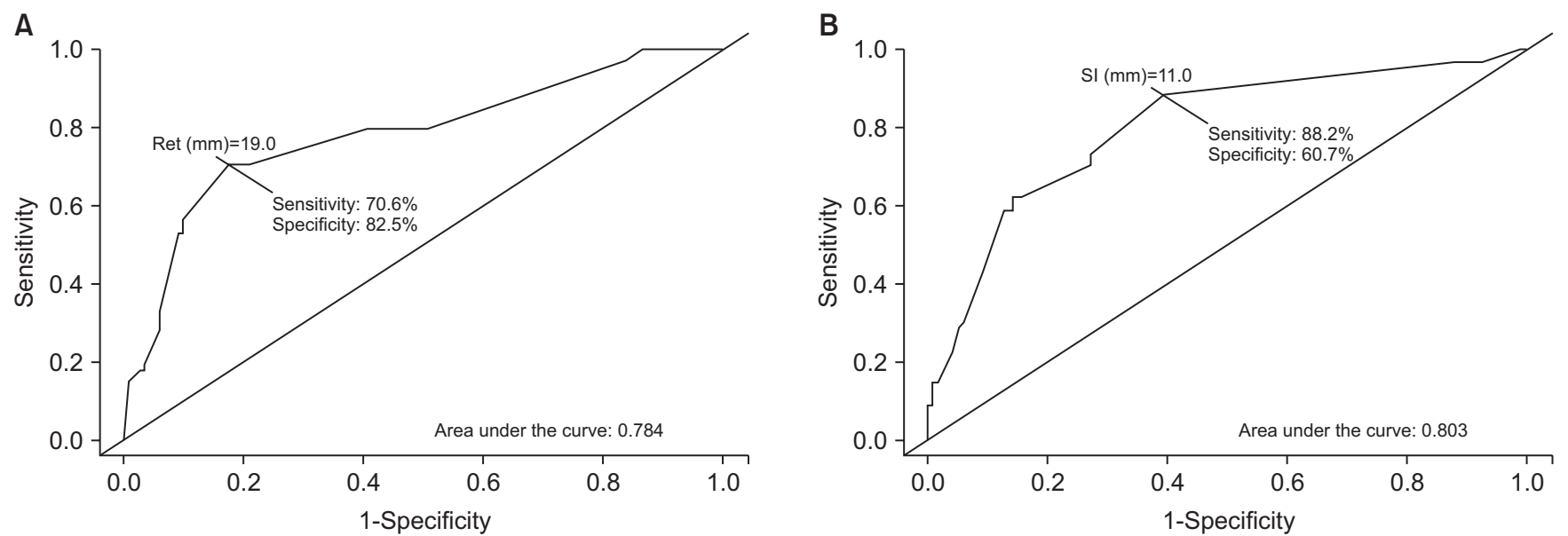

Fig. 2. Cut-off values for tear size for the prediction of subscapularis re-tears. (A) Ret: retraction, (B) SI: superior-inferior.

A
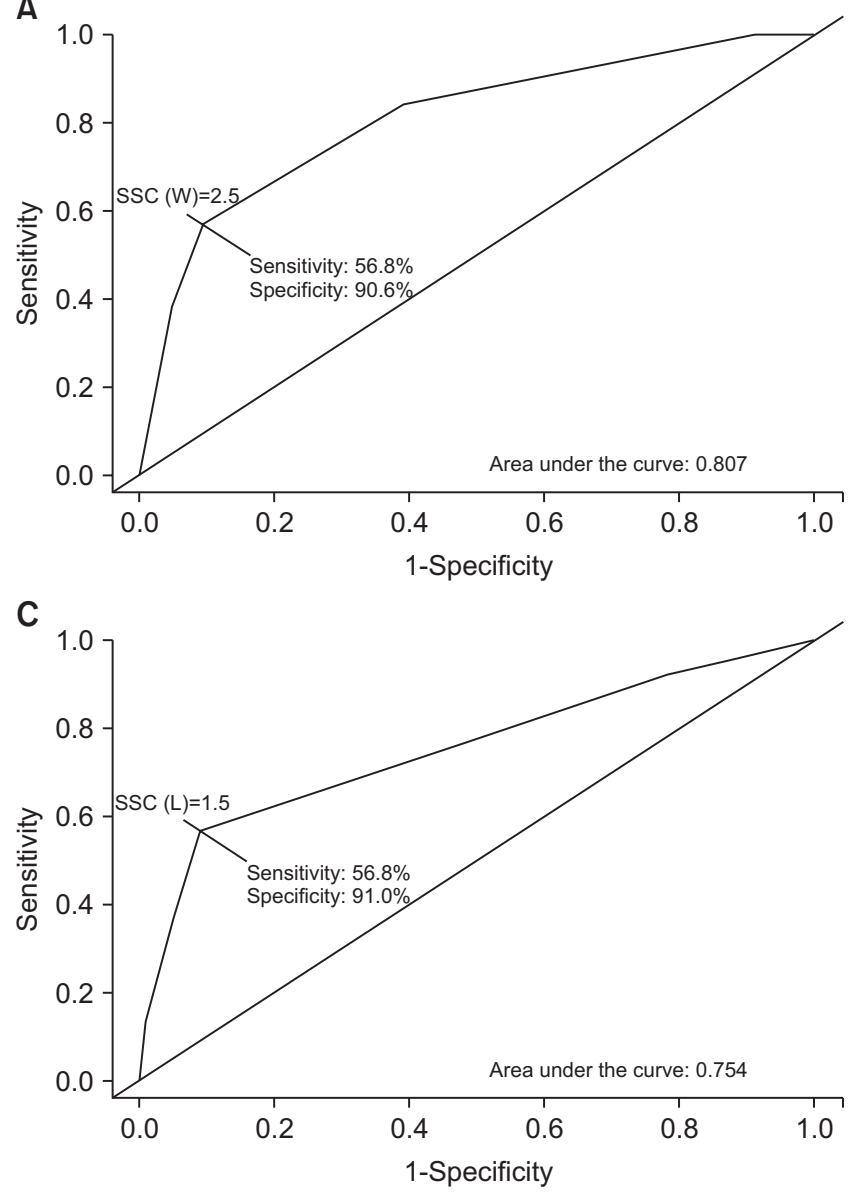

measured from upper cross-sections of the subscapularis may have a higher prognostic value than that measured from whole cross-sections. On the other hand, the cut-off value for fatty degeneration when it was quantified from lower cross-sections of the subscapularis was a Goutallier grade $1.5(\mathrm{A \cup C}=0.754)$, which was lower than when fatty degeneration was quantified

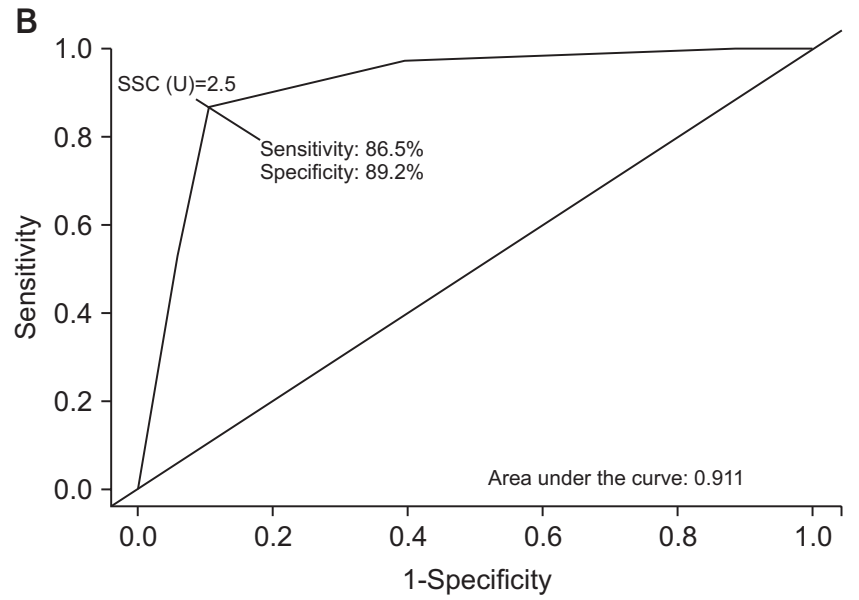

Fig. 3. Cut-off values for the degree of fatty degeneration by the area of subscapularis measured for the prediction of subscapularis re-tears. (A) SSC (W): subscapularis (whole cross-section), (B) SSC (U): subscapularis (upper crosssection), (C) SSC (L): subscapularis (lower cross-section).

from upper and whole cross-sections (Fig. 3).

\section{Discussion}

The degree of fatty degeneration has been shown to correlate with the size of subscapularis tear and the rate of postoperative 
re-tears in patients who underwent rotator cuff repairs involving the subscapularis. However, no studies have determined the superiority of quantifying the amount of fatty degeneration of the upper subscapularis over the whole subscapularis for the assessment clinical outcome and prognosis after cuff repairs. Here, our findings suggest that measuring the upper subscapularis only as opposed to the whole subscapularis, which requires the physician to measure up to the lower subscapularis, provides a reliable and easy way for physicians to predict prognosis at the clinical setting.

Studies that have compared the upper and lower subscapularis are mostly anatomical and functional studies. None have investigated whether the area of subscapularis taken to quantify the amount of fatty degeneration has an effect on the ability of the value quantified to predict clinical outcomes. Perhaps similar to the anatomical relationship between the upper and lower subscapularis muscles is that of the supraspinatus and the infraspinatus muscles. Studies in the past have attempted to determine the degree of fatty degeneration in the supraspinatus and the infraspinatus separately, but they have reported that the two structures are difficult to differentiate and that the predictive value of the quantified value was low. ${ }^{10)}$ In contrast, Seo et al. ${ }^{11)}$ reported that whether the fatty degeneration was measured from the upper infraspinatus or the lower infraspinatus, both quantifications showed a significant correlation with postoperative outcome measures such as tear size, function in terms of the ASES score, and pain. Interestingly, they showed that depending on the infraspinatus muscle the degree of fatty degeneration was measured from, the degree of fatty degeneration was associated with different ROMs: the degree of fatty degeneration of the upper infraspinatus correlated with abduction while the degree of fatty degeneration of the lower infraspinatus correlated with abduction and external rotation. Consistent with previous findings, our findings show that the ability to predict clinical outcome or prognosis does not improve with the area of subscapularis taken to quantify the amount of fatty degeneration - that is, the amount of fatty degeneration of the upper subscapularis was not any more predictive of clinical outcome than that of the whole subscapularis. If we had seen that the former approach led to a statistically significant correlation but the latter approach did not, then we would have been able to argue that fatty degeneration can serve as an indicator of clinical outcome after cuff repairs only when the amount of fatty degeneration is measured from the upper subscapularis and not from the lower or whole subscapularis. However, we found that irrespective of the degree of fatty degeneration such as tear type, history of trauma, or the two approaches revealed a similar level of correlation between fatty degeneration and our measures of clinical outcome, tear size and re-tear rate. These findings imply that our results alone cannot be used to argue the superiority of one approach over the other.

We found that a history of trauma did not show an association with the degree of fatty degeneration for all quantification methods. But patients with a history of trauma were more likely to have isolated tears than those who did not have a history of trauma (61.9\% vs. $26.7 \%)$. However, because the number of patients with isolated subscapularis tears was extremely low (6.7\%), this correlation between trauma history and fatty degeneration should be corroborated through studies with larger sample populations.

Previously, studies have shown that the upper and lower subscapularis are not only controlled by different nerves ${ }^{3)}$ but also responsible for different movements. ${ }^{3,12)}$ Like previous authors, ${ }^{11)}$ we hypothesized that the association between the degree of fatty degeneration and clinical outcomes such as internal rotator strength would differ by the area of subscapularis measured to quantify the degree of fatty degeneration. Here, we found that the inverse association between fatty degeneration and internal rotator strength did not significantly differ by the area of subscapularis quantified. This may be because unlike the infraspinatus, which predominantly controls abduction and external rotation, the subscapularis controls internal rotation, which can also be controlled by, for instance, the pectoralis major or latissimus dorsi muscle, which act complementary to the subscapularis as an alternative source of internal rotator strength.

We found that tear size showed a strong correlation with the degree of fatty degeneration, but measuring fatty degeneration from only the upper subscapularis was not any more correlated with tear size than when it was measured from the whole subscapularis. Irrespective of the area of subscapularis measured, the size of tear was significantly associated with the level of fatty degeneration. Similarly, we found that the re-tear rate was positively correlated with the degree of fatty degeneration; this correlation was significant for when the upper and whole crosssections of the subscapularis were taken. We found that the former approach was not superior over the latter approach for any of the other parameters.

Although the degree of fatty degeneration was on average lower when the lower subscapularis was measured than when the upper subscapularis was measured (average Goutallier grades 1.01 vs. 1.71), measuring fatty degeneration from the whole subscapularis (average Goutallier grade 1.59) could reflect significant correlation with clinical outcomes. When we consider the ease of measurement, it is more important for the physician to be able to discriminate visually large areas of fatty degeneration rather than to discriminate small degenerative changes. Therefore, the findings of our study, which we derived by assessing the correlation between fatty degeneration and clinical outcome in only patients with re-tears, has an important clinical implications: the approach to quantify fatty degeneration of only 
the upper subscapularis can be used as a means for physicians to predict the possibility of re-tears with greater ease. We divided the degree of fatty degeneration by whether they encompassed more (severe) or less than half (mild) of the subscapularis (either Goutallier grades $0-2$ or Goutallier grade 3-4, respectively). Interestingly, we found that when only upper cross-sections of the subscapularis were used to quantify the degree of fatty degeneration there was a significantly higher proportion of patients with severe fatty degeneration than when whole cross-sections were used $(86.5 \%$ vs. $56.8 \%, p<0.05)$. Although both approaches to measure fatty degeneration are useful to predict for re-tears, the ease of identification for physician is probably higher in the measuring fatty degeneration of the upper subscapularis. When fatty degeneration was measured from the lower subscapularis only, we found that a greater proportion of patients with re-tears had mild fatty degeneration (78.4\%) than severe fatty degeneration. This finding suggests that fatty degeneration measured from the lower subscapularis has little value as a predictor for re-tears. Moreover, we found that the cut off value for fatty degeneration, our predictor of postoperative re-tears, was the same for the upper subscapularis- and the whole subscapularis-derived quantifications of fatty degeneration, but a higher AUC for the former approach implies a higher predictive power for postoperative retears.

Our findings highlighting new relationships between the degree of fatty degeneration, tear size, and re-tear rates by the area of subscapularis measured suggest important clinical implications. Although we could not observe a significant correlation between internal rotator strength and the degree of fatty degeneration, we reason that this may be because we measured internal rotation only at $0^{\circ}$ of abduction. Previous studies have shown that internal rotator strength measured at $90^{\circ}$ of abduction and $90^{\circ}$ of external rotation showed a strong correlation with lesions of the upper subscapularis. ${ }^{13)}$ Moreover, certain tests, such as the lift-off, belly-press, and bear-hug tests, have each been correlated with different parts of the subscapularis. ${ }^{14,15)}$ These findings suggest that future studies may investigate correlation the distinction between the upper and the lower subscapularis for the measurement of fatty degeneration with the measurement of internal rotator strength in varying positions.

\section{Conclusion}

We found that the degree of fatty degeneration significantly correlated with subscapularis tear size and the rate of re-tears, but we could not demonstrate the superiority of quantifying the degree of fatty degeneration of only the upper cross-section of the subscapularis over the whole cross-section for the predictive value of the fatty degeneration. Nevertheless, we suggest that quantification of the upper cross-section only as opposed to the whole subscapularis has clinical significance because it provides an easier and a more practical way for surgeons to assess for retears and prognosis at the clinic during postoperative follow-ups.

\section{References}

1. Clark JM, Harryman DT 2nd. Tendons, ligaments, and capsule of the rotator cuff. Gross and microscopic anatomy. J Bone Joint Surg Am. 1992;74(5):713-25.

2. Kenn W, Böhm D, Gohlke F, Hümmer C, Köstler H, Hahn D. 2D SPLASH: a new method to determine the fatty infiltration of the rotator cuff muscles. Eur Radiol. 2004;14(12):2331-6.

3. Decker MJ, Tokish JM, Ellis HB, Torry MR, Hawkins RJ. Subscapularis muscle activity during selected rehabilitation exercises. Am J Sports Med. 2003;31(1):126-34.

4. Jeong JY, Pan HL, Song SY, Lee SM, Yoo JC. Arthroscopic subscapularis repair using single-row mattress suture technique: clinical results and structural integrity. J Shoulder Elbow Surg. 2018;27(4):711-9.

5. Longo UG, Berton A, Marinozzi A, Maffulli N, Denaro V. Subscapularis tears. Med Sport Sci. 2012;57:114-21.

6. Warner JJ, Higgins L, Parsons IM 4th, Dowdy P. Diagnosis and treatment of anterosuperior rotator cuff tears. J Shoulder Elbow Surg. 2001;10(1):37-46.

7. Lee YS, Jeong JY, Park CD, Kang SG, Yoo JC. Evaluation of the risk factors for a rotator cuff retear after repair surgery. Am J Sports Med. 2017;45(8):1755-61.

8. Goutallier D, Postel JM, Gleyze P, Leguilloux P, Van Driessche S. Influence of cuff muscle fatty degeneration on anatomic and functional outcomes after simple suture of full-thickness tears. J Shoulder Elbow Surg. 2003;12(6):550-4.

9. Pfirrmann CW, Schmid MR, Zanetti M, Jost B, Gerber C, Hodler J. Assessment of fat content in supraspinatus muscle with proton MR spectroscopy in asymptomatic volunteers and patients with supraspinatus tendon lesions. Radiology. 2004;232(3):709-15.

10. Zanetti M, Gerber C, Hodler J. Quantitative assessment of the muscles of the rotator cuff with magnetic resonance imaging. Invest Radiol. 1998;33(3):163-70.

11. Seo JB, Yoo JS, Jang HS, Kim JS. Correlation of clinical symptoms and function with fatty degeneration of infraspinatus in rotator cuff tear. Knee Surg Sports Traumatol Arthrosc. 2015; 23(5):1481-8.

12. Digiovine NM, Jobe FW, Pink M, Perry J. An electromyographic analysis of the upper extremity in pitching. J Shoulder Elbow Surg. 1992;1(1):15-25.

13. Lin L, Yan H, Xiao J, Ao Y, Cui G. Internal rotation resistance test at abduction and external rotation: a new clinical test for diagnosing subscapularis lesions. Knee Surg Sports Traumatol Arthrosc. 2015;23(4):1247-52. 
14. Chao S, Thomas S, Yucha D, Kelly JD 4th, Driban J, Swanik K. An electromyographic assessment of the "bear hug": an examination for the evaluation of the subscapularis muscle. Arthroscopy. 2008;24(11):1265-70.
15. Tokish JM, Decker MJ, Ellis HB, Torry MR, Hawkins RJ. The belly-press test for the physical examination of the subscapularis muscle: electromyographic validation and comparison to the lift-off test. J Shoulder Elbow Surg. 2003;12(5):427-30. 